\title{
Le harpon et le bâton (Joola-Felup, Guinée-Bissau)
}

The Harpoon and the Stick (Dyola-Felup, Guinea-Bissau)

Odile Journet

\section{OpenEdition}

Journals

Édition électronique

URL : http://journals.openedition.org/span/1308

DOI : $10.4000 /$ span. 1308

ISSN : 2268-1558

\section{Éditeur}

École pratique des hautes études. Sciences humaines

\section{Édition imprimée}

Date de publication : 1 novembre 1993

Pagination : 17-38

ISSN : 0294-7080

\section{Référence électronique}

Odile Journet, « Le harpon et le bâton (Joola-Felup, Guinée-Bissau) », Systèmes de pensée en Afrique noire [En ligne], 12 | 1993, mis en ligne le 03 décembre 2013, consulté le 01 mai 2019. URL : http:// journals.openedition.org/span/1308; DOI : 10.4000/span.1308 


\section{LE HARPON ET LE BATON \\ (Joola-Felup, Guinée-Bissau)}

par

\section{Odile Journet}

Parler d'"objet-fétiche" à propos d'un bëkin (pl. ukin) joola (ou l'un de ses éléments) m'a longtemps paru problématique. Non en raison des difficultés théoriques ou idéologiques que pourrait soulever la définition du terme "fétiche", mais à cause de la diversité et de la polysémie des matériaux de terrain. Si, pour les Joola-Felup du nord de la Guinée-Bissau', pratiquer les cultes des ukin, c'est être enayaw ("sacrifice") - par opposition au christianisme ou à l'islam -, cette référence ne permet pas pour autant de définir un champ de pratiques homogènes.

Bëkinabu est le terme utilisé pour désigner tout à la fois le sanctuaire, l'autel ("la bouche" du bëkin), et la puissance propitiée, laquelle peut prendre l'apparence d'un personnage anthropomorphe: les responsables de culte le décrivent alors comme un être "court", de la taille d'un enfant de dix ans, mais extrêmement fort et d'une carrure impressionnante, blanc ou noir. On peut le rencontrer en brousse, et lors des

${ }^{1}$ Les données auxquelles nous nous référons ici ont été recueillies lors de récentes enquêtes menées par A. Julliard et moi-mêrne dans le village de Susana (région de Cacheu).

Fétiches II. Puissance des objets, charme des mots, Systèmes de pensée en Afrique noire, 12, 1993 
sacrifices, invisible aux yeux des participants, il est là, assis près de l'autel. Le bëkin peut également apparaître sous la forme d'un serpent. Médiateur entre Emitay, Maître du ciel, des saisons et de la pluie, et les humains, chaque bëkin est doté d'attributions spécifiques: initiation, accouchement, vengeance, guérison, traitement du meurtre ou du vol...

Sur l'origine des ukin, les Joola ne sont en général guère prolixes. La plupart s'accordent à dire que "le bëkin vient de la mer" (d'où la présence d'objets marins, coques et coquillages, dans les sanctuaires et la parure des responsables). L'idée que la mer soit le siège de puissances mythiques redoutables est largement répandue dans toutes les populations côtières du Sénégal et de Guinée-Bissau²: un mythe joola fait allusion au serpent python ejunfur qui, une fois qu'il a atteint une certaine taille, sort d'une mare pour se mesurer à un rônier. Lorsqu'il parvient au sommet, il se met à pleuvoir. Il se transforme alors en taureau et se dirige vers la mer. Sorti de cet univers aquatique, le bëkin réside au plus profond de la forêt, où il vit seul ou en famille. Mais les détenteurs de culte diront aussi que le bëkin siège "dans la terre", dans ce milieu souterrain auquel est fortement lié le destin des habitants. Espace invisible chargé de puissances, etamay désigne à la fois le sol, le champ cultivé, le territoire, et les habitants qui en sont originaires. Maintes expressions en font un usage synecdochique pour parler des ukin (etamay afagnut: "la terre refuse"; utyametam "payer la terre", etc.).

Etre de la mer, être de la terre, le bëkin, sur ordre de Emitay, gouverne également les vents, bons ou mauvais, qui apportent la santé ou la mort au village. Pour conserver sa force (sembe, force au sens physique du terme), un bëkin doit être régulièrement arrosé de sang ou de vin de palme (la sève de l'arbre et le sang animal étant désignés par le même terme: asimew). Il incombe à son responsable de gérer adroitement cette force, afin de ne pas la disperser: ainsi, pendant les jours qui précèdent les luttes, est-il impossible de sacrifier pour d'autres causes à certains ukin, accaparés par le travail qu'ils auront à fournir auprès des grands lutteurs. "On ne peut pas demander trop de choses

2 Les Añaki (groupe Bijogo) appellent elamundi une puissance dont l'origine est un vieux python qui est allé vivre dans la mer (Ch. Henry, 1991: 122-123). 
a la fois au bëkin, il ne pourrait rien faire de bon". De son intervention, il est par contre bien difficile de maîtriser le moment précis: il arrive qu'il agisse trop tôt ou trop tard ${ }^{3}$.

Sous son aspect d'autel ou de sanctuaire, le bëkin n'est guère comparable à des objets tels que par exemple, les boli ou les yapèrè de l'aire mandé, que l'on sort, que l'on porte, que l'on crépit. Il évoquerait plus les pangol serer ou les tuur wolof et lebu: une fois "planté" (le même terme, kagiten, désigne l'action de planter un arbre et de planter un bëkin), il est fortement enraciné dans le sol. On ne le déménage qu'en de très rares occasions, et si etamay (la terre) le veut bien. Un bëkin sera ainsi "planté" dans la chambre d'une maison, dont la porte et le plafond sont particulièrement bas, dans une case particulière, ou encore en plein air, au milieu d'un bosquet (à l'intérieur ou à proximité du village), au pied d'un gros arbre, ou en forêt. L'élément central, l'"autel", que l'on arrose de vin de palme, de sang, ou d'eau, est constitué d'un trou creusé, ou d'un pieu fourchu. Une ou plusieurs poteries, parfois des feuilles de tabac, des objets liés au domaine traité, peuvent être enterrés sous le sol. Au fur et à mesure des années et des sacrifices, le bëkin s'enrichit des mâchoires et des cornes des animaux sacrifiés, ainsi que des instruments rituels (poteries et coquillages de libation en particulier) de ses détenteurs défunts. Ces reliques et objets consacrés au bëkin, et en quelque sorte absorbés par lui, ne sont pas simples décorations, comme le montre l'anecdote suivante. Un matin de janvier 1990, menacé par l'incendie d'une maison proche, bulampanabu, bëkin de protection générale, l'un des plus importants du village, faillit brûler. Tandis que les hommes du quartier grimpés sur la toiture s'efforçaient d'éteindre les flammèches, Wagam, le responsable, restait obstinément assis dans son bëkin. "Si le bëkin doit brûler, je préfère brûler avec". Il nous expliqua par la suite qu'en cas de destruction matérielle du sanctuaire, il aurait dû réitérer tous les sacrifices accomplis depuis l'installation du bëkin et

${ }^{3}$ De l'analyse minutieuse qu'il faisait de la religion joola, L.V. Thomas (1959) retenait comme l'une des dimensions principales, la représentation d'un univers comme "sièges de multiples déplacements de forces nourries et nourrissantes" qu'il qualifiait de "pneumatisme". 
remplacer tous les objets de culte. Tâche impossible lorsque l'on considère la composition de bulampanabu. Installé sous la véranda arrière de la maison, sur une longueur d'environ 8 mètres, il a pour autel principal un trou creusé dans le sol, entouré d'un amas de gros coquillages et de quatre piquets de bois fichés en terre, eux-mêmes coiffés d'une coquille où sont piquées des plumes de coqs. Posés à terre face à l'autel, de longs troncs équarris servent de bancs aux participants. Le mur est entièrement tapissé de milliers de mâchoires et de plus d'une centaine de cornes d'animaux sacrifiess. Sur toute la longueur du sanctuaire sont suspendues les mâchoires qui n'ont pu trouver place sur le mur, et des gerbes de riz. A l'entrée de la véran$\mathrm{da}$, de vieux harpons sont appuyés; deux hochets-calebasse sont accrochés au milieu des mâchoires. Dans le fond sont également entreposés d'imposants canaris exclusivement réservés à la cuisine des grands sacrifices. La véranda permet d'accéder à un enclos qui la jouxte, entourée d'une clôture de bois rouge, à l'intérieur de laquelle est érigé un deuxième autel (un pieu fourchu) où s'effectue la mise à mort des porcs. Dépôt des archives du sacrifice dans les domaines qu'il régit, bulampanabu pourrait également être comparé à une sorte de registre d'état civil: à la naissance de chaque enfant du village a été "plantée" sous terre une petite poterie (ebegey), où l'on a effectué le sacrifice d'un æuf, et quelque temps plus tard, d'un porc. Ces poteries dont le col affleure parfois à la surface sont rangées par lignage et par ordre de naissance. Le responsable doit savoir retrouver chacune d'elles lors de sacrifices ultérieurs, à la demande de tel ou tel natif du village (plus d'un millier et demi dans le cas présent).

Tous les sanctuaires ne sont pas aussi imposants: certains, plus récents, ou moins fréquentés, simples "annexes" d'un grand bëkin, se réduisent aux éléments qui composent l'autel, devant lequel une petite place a été aménagée. Un village peut compter une bonne centaine, parfois plus, de ces sanctuaires, que l'on peut ordonner dans un premier temps en fonction des filiations existant d'une part entre bëkin "mère" et bëkin "enfant" et, de l'autre, entre le sanctuaire principal et ses multiples annexes.

L'espace social et religieux que définit l'ensemble complexe des $u k i n$ laisse apparaître, dans la pratique rituelle, une première ligne de 
séparation fondée sur la dualité des sexes: en résumé, tout ce qui se rapporte aux initiations, à la royauté, au droit villageois, à l'histoire lignagère, est sous la tutelle d'ukin réservés aux hommes; tout ce qui touche à la procréation et à la fertilité dépend des ukin réservés aux femmes. Dans un précédent article (Journet, 1987), j'avais tenté d'explorer les implications de cette division. L'analyse du partage des compétences respectivement attribuées à tel ou tel bëkin laissait cependant dans l'ombre un certain nombre de questions, parmi lesquelles celle du lien qui unit le (la) desservant(e) à son bëkin.

\section{Les deux voies d'accès à la fonction de amañen}

Le détenteur d'un bëkin est appelé "amañen", ou bien "ata" ("maître", "propriétaire") suivi du nom de son bëkin, ou encore "ahaan" ou "anare-bëkin" ("homme" ou "femme" du bëkin). Un dicton rappelle les risques que comporte sa position: "la tête rasée ne poussera pas" (celui qui prend indûment un bëkin mourra avant que ses cheveux, rasés lors de son intronisation, ne repoussent).

Il est deux façons de devenir amañen. La première consiste en une importante tournée sacrificielle, dite ulangaw. Cette démarche personnelle du (de la) futur(e) responsable n'est pas pour autant délibérée. Le bëkin, sous son apparence anthropomorphe ou animale, lui est apparu, lors d'un déplacement solitaire en brousse, ou lors d'un rêve. Ou bien, il l'a "saisi", dans une crise, un évanouissement, une maladie spécifique; il s'acharne à le tourmenter par des ennuis répétés. Une consultation divinatoire va indiquer quel est le bẻkin qui réclame ainsi son amanten. C'est donc par une tournée sacrificielle particulièrement fournie en sang et en vin de palme que le futur détenteur attirera le bëkin génie dans le sanctuaire qu'il va reprendre ou planter auprès de sa maison.

Dans cette société de riziculteurs, faire le ulangaw est une affaire longue et coûteuse, du fait de la quantité de sacrifices exigés: au minimum un chien, cinq porcs, un bœuf, une chevre, de nombreux poulets et une ou plusieurs centaines de litres de vin de palme. De 
plus, le candidat doit nourrir tout le quartier pendant une semaine joola (6 jours). S'y dérober trop longtemps, c'est se mettre en danger de mort: c'est ce que révelent un certain nombre d'interrogatoires de cadavres de personnes décédées dans leur âge mûr. Le ulangaw permet de reprendre un bëkin déjà "planté" ou de fonder un nouveau sanctuaire (inséré toutefois dans une chaîne de filiation d'ukin préexistants). Certains responsables (il ne s'agit alors que d'hommes ${ }^{4}$ ) peuvent être appelés a faire le "grand ulangaw" - comportant entre autres le sacrifice de cinq taureaux - qui leur permet de sacrifier et d'organiser les rituels dans tous les ukin acquis de cette façon.

Le champ d'intervention et les domaines traités par ces ukin sont multiples: protection, divination, vol, meurtre, vengeance, feu, chutes, maladies, folie, blessures, piqûres de serpent, etc.

La deuxième manière est plus radicale: c'est le rapt (usohi). Le candidat choisi est capturé à l'improviste par les conseillers et adjoints au bëkin lors des fêtes calendaires de esangey (pour les hommes) et de karaayaku (pour les femmes), qui, tous les six ans, ponctuent l'intervalle entre deux rituels initiatiques. A cette occasion, seront pourvus tous les sanctuaires laissés vacants par la mort de leur détenteur. Il s'agit pour l'essentiel des ukin liés à l'initiation masculine (karenaku ou akuyu), à la maternité (karaayaku), à la royauté (këyëku). Ces ukin se transmettent à l'intérieur d'un, ou alternativement, de deux lignages $^{5}$, mais très généralement, nul ne tient à en assumer la charge (qui comporte, entre autres contraintes, une assignation à résidence définitive). Le usohi donne fréquemment lieu à des situations tragiques ou rocambolesques, les éventuels pressentis, particulièrement ceux qui ont émigré en ville ou dans d'autres régions, cherchant à échapper à ceux qui viennent les enlever. Mais ils peuvent tout aussi bien être pris dans un village voisin. Quoi qu'il en soit, nul sacrifice personnel n'est exigé de l'intronisé; une fois capturé, il est emmené dans la forêt où est planté le bëkin "mère", et initié à ses fonctions. A son retour au village, il reste reclus pendant cinq jours dans une hutte. Peu importe

4 A titre indicatif, 8 hommes de ce village de 2000 habitants environ ont fait le grand ulangaw.

${ }^{5} \mathrm{Si}$ l'on ne trouve personne dans le patrilignage, on ira chercher le neveu utérin. 
son âge, il suffit qu'il/elle soit déjà marié/e, qu'il ait été initié s'il s'agit d'un homme, qu'elle ait eu un enfant s'il s'agit d'une femme.

A ces deux voies d'accès à la responsabilité du bëkin, correspondent deux attributs bien différents: le harpon (ujokosahu) dans le premier cas, le bâton (utampangahu) dans le deuxième.

Posséder le harpon, c'est aussi être guérisseur (kabonen: soigner) et dépositaire de la connaissance des plantes médicinales.

\section{Deux séries d'objets :}

Objets que nous avions initialement considérés comme simples attributs emblématiques du statut d'amañen, et des manières de l'acquérir, le harpon et le bâton ont un statut spécifique et participent de procès rituels bien différents. Nous essaierons de voir en quoi leur opposition correspond à deux types de pratiques, à deux modes de relation au bëkin, opposition que l'on ne peut réduire ni à la division fonctionnelle, ni à une quelconque hiérarchie des cultes. Arrêtons-nous un instant sur ces objets.

\section{Le harpon.}

Pourquoi un harpon dans une population dont toutes les attentions matérielles et rituelles sont orientées vers la pratique de la riziculture ? Nous pensions avoir un élément de réponse dans l'idée énoncée plus haut que "le bëkin vient de la mer". Mais à nos questions, les gens du ulangaw ont longtemps opposé des réponses dilatoires. Ce sont des affaires de doubles et de chasse racontées en d'autres occasions qui nous ont permis de mieux comprendre son rôle rituel. Le harpon, le long duquel sont enfiles de petits anneaux de fer, est rarement utilisé comme arme: les pêcheurs de la côte (Niominka pour la plupart) s'en servent pour la pêche aux très gros poissons (requins par exemple); les Felup n'y ont recours que pour la chasse à l'hippopotame (eñogay). Tous animaux qui, dans les conceptions joola de la personne, sont considérés comme des "doubles" (ewumey) d'êtres humains vivants. Né en brousse ou dans l'eau, quelques heures ou quelques jours avant la 
naissance d'un enfant, le double animal suit la destinée de son homologue humain ${ }^{6}$. Si toute personne n'a pas pour double un hippopotame, tout hippopotame par contre est un double. Aussi la mise à mort de cet animal est-elle rituellement traitée comme un meurtre: le premier chasseur à l'avoir atteint doit sacrifier un bouf au bëkin akuyu (initiation des hommes) et un porc au karaayaku des femmes. En l'honneur du double animal, les villageois exécutent les danses funéraires nyukulay. Et l'on s'attend à un décès humain dans les prochains jours.

Tandis que la lance (ejaney) est réservée au combat contre des êtres - humains ou animaux - visibles, il apparaît que le harpon est utilisé chaque fois qu'est mise en jeu l'une des dimensions occultes constituantes d'une personne : utilisé en cas de force majeure contre un "double", il est également l'arme privilégiée de la lutte contre les sorciers asayo (de type "witch"). Selon les théories felup, certaines maladies sont liées au fait qu'une "personne mauvaise", "dont la tête est sale"..., s'est emparée de votre "cœur" (yalorey: principe qui n'est pas confondu avec l'organe du cœur, mais qui y siège), afin de le transformer en animal et de le dévorer. C'est avec son harpon, précédé du cliquetis de ses petits anneaux de fer, que l'amanen va attaquer l'agresseur pour récupérer ce "cour" volé. Ou bien on apprendra que tel amañen, décidé d'en finir avec un sorcier depuis longtemps suspecté, est parti en pleine nuit piquer de son harpon le tronc d'un gros fromager. Et l'on découvrira au pied de l'arbre une branche cassée, dévoilant à l'endroit de sa brisure, des cuillers et de l'eau qui coule. Le sorcier qui venait s'y cacher pour manger, meurt le lendemain.

Dans ces combats, le harpon "pique" ou bien, vient se ficher en terre devant la personne visée, lui liant les genoux et la paralysant.

Le harpon d'un responsable décédé ne peut être transmis qu'à l'un de ses frères (de même père). Sinon, le nouvel amañen doit apporter du fer au forgeron du quartier (la région ne possédant pas de minerai, il s'agit là d'une quête difficile) et acquitter d'importants sacrifices au bëkin de la forge.

${ }^{6}$ La croyance au double animal, sous une autre variante, a été étudiée dans une région du nord-est de la Basse-Casamance par J. David Sapir (1977). 
Le harpon est par ailleurs entièrement soumis aux interdits de contact avec les lieux ou les personnes qui doivent rester rigoureusement séparés du bëkin qu'il représente: il peut arriver que son détenteur, s'il s'agit d'un homme, soit amené à entrer dans une maison où se trouve une femme en règles, ou nouvellement accouchée. Il doit alors laisser son harpon au dehors (un amañen "à bâton" resterait luimême au-dehors).

Les détenteurs de harpon possèdent également une série d'objets: . le grand coquillage epatey (de type conus papilionaceus) que portent en collier, ou en bandoulière, ceux qui ont fait le grand ulangaw: il frappe à la tête ou tombe devant celui qui oserait s'attaquer aux protégés du bëkin et le paralyse. Dans cette fonction, epatey est inséparable de la corde ebambuyèmube, sur laquelle il est enfilé.

. le hochet-calebasse esewuey: il attaque les personnes animées de mauvaises intentions en "leur prenant la tête"; celle-ci se met à tourner et à résonner comme le hochet, provoquant la folie.

- sans posséder à proprement parler d'efficacité opérationnelle, les bracelets de fer (banenlenkabu) portés par les détenteurs de harpon, symbolisent au moins deux choses: l'invulnérabilité de l'amanen, et l'action du bëkin. Les bracelets de l'amañen sont "comme ceux que portent les lutteurs" pour empêcher leur adversaire de les saisir par le poignet. Cercles fermés, ils évoquent le travail du bëkin qui tout à la fois "englobe", "enserre", "termine" une affaire. Scandée par les coups sourds de la coquille de libation frappée au sol, écoutons à ce propos l'adresse initiale faite à bulampanabu par son amanten :

"jiigohen! ("celui qui couvre", comme la poule étend ses ailes sur ses poussins)

"janten! ("il faut écouter")

"arambaasekol! ("femme de la brousse")

"jilinken ! ("celui qui ferme" - comme on ferme un trou, une tombe, une parole, comme on "finit une affaire")

"akilesuk! ("le maître du village")

"atiika! ("le guerrier")

"jilomen! ("celui qui enserre", comme on enserre avec les doigts le bëkin qui "prend", on ne peut lui échapper)." 
Les cliquetis clairs des harpons et les crissements plus sourds des hochets-calebasse ont également valeur d'avertissement, et traitent préventivement l'espace où pourraient éclater bagarres, discussions et règlements de comptes ${ }^{7}$. Ainsi, quelques jours avant les grandes luttes villageoises, les "grands" du ulangaw parcourent-ils les sentiers qui délimitent chaque quartier pour en écarter toute mauvaise intention. De même avant les combats de chaque classe d'âge feront-ils le tour de l'aire de lutte pour prévenir les incidents qu'un climat souvent fort tendu laisse présager ${ }^{8}$.

Les gens du ulangaw détiennent fréquemment des ukin de divination: dans ce cas, les techniques utilisées (corne, tambour a friction, petite natte, pendule, etc.) sont particulières à chaque responsable et peuvent être, au gré de chacun, perfectionnées ou empruntées à l'extérieur.

\section{Le bâton}

Le bâton que portent les hommes détenteurs des ukin de karenaku (initiation), ou këyëku (royauté) est un bâton de bois rouge, taillé dans du caïlcédrat (dit "bois de fer"), d'une longueur d'environ 1,50 m, et agrémenté à ses deux bouts d'un capuchon de cuivre. Confié au responsable de culte lors de son intronisation, il est élément du bëkin, au même titre que la grande coque kasendaku (de type cymbium neptuni) qui sert aux libations de vin et de sang, ou encore le petit tabouret royal erumburumay. Seuls leurs propriétaires peuvent les porter ou les toucher. Pour les femmes, il s'agit plus généralement d'un bâton plus court, souvent recouvert de rangs de perles, qu'elles portent en bandoulière. Sur le détail de la taille de ces bâtons, nous n'avons guère d'informations, si ce n'est qu'il est remis au responsable après avoir

7 Elobey (de elob: parler): "discussion", "affaire", "vengeance"...

${ }^{8}$ Lors des luttes organisées par les responsables de bulampanabu ou këyëku, plus de 25 villages, entre lesquels les problèmes d'appropriation de rizières sont permanents, peuvent se confronter. Si une bagarre éclate, l'amañen en est responsable devant son bëkin et doit sacrifier du vin. Si le sang coule, il devra immoler un boeuf dans son sanctuaire. 
été "arrosé" de vin et de sang, et qu'il le sera régulièrement lors de sacrifices ultérieurs.

Insignes et parties intégrantes du bëkin, ces objets, à la différence de la série précédente, n'ont pas de mode d'action spécifique. Leurs détenteurs ne soignent ni ne prescrivent. Ils ne manipulent pas leurs attributs comme armes magiques lors de combats nocturnes. Alors que leurs homologues "a harpon", en sus du sacrifice, consultent leur bëkin pour des prescriptions et mènent, grâce à leurs instruments rituels, une guerre invisible contre les agresseurs de leurs consultants, les responsables "à bâton" ne peuvent qu'entendre les confessions (jarèjaju) de celui ou celle qui a transgressé les règles du bëkin, et sacrifier. Dépositaires de la force du bëkin, ils ne peuvent la diriger. L'efficacité attribuée au petit balai royal (ullasaw), constitué d'un faisceau de pailles, semblerait contredire cette opposition: il peut en effet faire tomber tout ce qu'il touche sous la coupe du bëkin këyëku, qu'il s'agisse de femmes, de riz ou de bétail. Ce pouvoir toutefois n'est autre que la projection du pouvoir même du bëkin, force d'attraction qui, telle un aimant, capte et incorpore tout ce qui releve des domaines qu'il traite. Ainsi les restes d'une maison brûlée sont-ils transportés à sambunasu, les grands canaris qui ont servi à apporter l'eau du bain de l'accouchée et du nouveau-né s'accumulent dans erumuney, les placentas dans le sol de karaayaku, les excréments des initiés dans ekobey, les bracelets des féticheurs défunts dans katolaku, et, dans chaque bëkin, cornes, mâchoires, plumes, coquillages de libation... Tous objets ou substances devenus par là-même nini (interdit, dangereux) en tant que constituants du bëkin.

\section{Deux champs}

L'examen du tableau des pages suivantes, répertoriant les principaux ukin d'un quartier felup, nous amène à formuler un certain nombre d'observations: a la différence des ukin "a harpon", les ukin "à bâton" ne tolèrent pas l'indifferenciation sexuelle. Ils fonctionnent sur le mode de l'exclusion réciproque, autant dans les domaines traités (l'initiation, "affaire des hommes" / la fertilité et l'accouchement, 
"affaire des femmes") que dans la pratique sacrificielle. Dans les sociétés joola, initiation masculine et accouchement sont les référents ultimes de l'identité adulte et de la différence sexuelle. Les deux tirent leur sens d'un ensemble de spéculations sur l'écoulement de sang (opposant les écoulements provoqués et les écoulements subis). Les interdits liés à ces deux types d'événement sont au premier chef des interdits d'ordre scopique, et corollairement d'ordre cognitif: d'avoir aperçu des circoncis dans la forêt, une femme peut mourir; il en est de même pour un homme qui aurait vu une femme accoucher. A l'initiation masculine est également associé un autre interdit: tandis que les femmes et les non-initiés doivent déféquer en brousse, les initiés ne peuvent le faire que dans l'enceinte du bëkin ekobey et de ses annexes. Toutefois, les afflictions provoquées par les ukin qui garantissent cette séparation n'ont pas trait, comme on serait tenté de l'augurer, à la vision, mais consistent en dysfonctionnements d'organes internes: tandis que les ukin réservés aux femmes frappent "au ventre", le "mal aux côtes" produit par les ukin liés à l'initiation est l'expression d'un grave trouble de la respiration, l'une et l'autre maladie engendrant déperdition et appauvrissement du sang. Diffuses et indéfinissables au regard de la précision des nosographies livrées par les guérisseurs "à harpon", mais d'autant plus mortelles, les attaques des ukin "à bâton" - "mal aux côtes", "maux de ventre" - ne se "soignent" pas par des médecines. Seuls la confession - anticipation préventive de l'interrogatoire du mort - et le sacrifice peuvent y remédier. La limitation des recours thérapeutiques semble ici faire écho a la rigidité du champ traite: car si les rituels d'initiation d'une part, les prescriptions et les représentations qui entourent l'accouchement d'autre part, construisent l'identité sexuelle, et les compétences attribuées à l'un et l'autre sexe, cette division ne tolère pas d'aménagements. Inscrite au cœur de la pensée classificatoire et de la pratique rituelle, référent primordial des règles du nini, elle ne peut donner prise à des opérations de transformation rituelle. Son analogon cosmique - la séparation et la succession des saisons sèche et pluvieuse, dont le roi, prêtre de këyëku, et les femmes de karaayaku sont garants dans leur personne physique - peut connaître des perturbations, mais ne peut être défait. Tout au plus est-il possible d'en différer les effets: retarder par exemple le moment de 


\section{Tournée sacrificielle}

rapt

Détenus par un homme exclusivement

Balampanabu " coiffe tous les ukin "à harpon" protection générale, maladies, luues

Kasentèku: prise de bëkin, ulangaw

Katafèku (3): chutes / mort d'enfants

Sambunasu: feu / lèpre

Kandongaku: forge / eczéma

Katolaku (3): enterrement / maux d'yeux

Ulinkinay: fonction de guérisseur

Jilibam : luttes / gale

Etamey: protection des lutteurs

Kanangenaku: chasse / rétention urinaire

Egulay: vol / problèmes d'articulations

Elenkiney: ? / hémorrö̈des

Kasaaraku: prédiction / vertiges / évanouissements

Balingabu: meurtre / folie

Ejenkay: vol / aphasie

Upinkaw: paralysie

Ujotongaw: surdité
Akuyu ou karer̃aku : initiation masculine (Bukut) / mal aux côtes

Ekobey (3): défécation des initiés

Jasang: circoncision

Ubiyew, kalukul, kupanduk, agongolaw: annexes de kareñaku

Këyëku: royauté / mort des enfants / plue

\section{Détenus par une femme exclusivement}

Egani-eganey: alliance / folie

Karaayaku: matemité, fécondité, séchen:sse, épidémies, invasion d'insectes / maux de ventres, coliques

Erumuney (3): accouchement

Karibile, ubiyèw (3): annexes de karaayaku

Eripay: opération des femmes mortes en couches

Ekungey: pluie

Détenus par un homme ou par une femme

Ankurenaw*(4): divination / maux de tête

Amumew*(3): divination / maux de tête

Buluntabu: jumeaux / enflements du corps

Kanewaku: alliance / diarthées enfant

Emotay: vol / articulations, maux de dents

Bulukabu: piqûres de serpent, blessures par bris de canari, déboîtements, fractures

Ugonkaw: vol / maux de reins / marche des enfants

Unijaw: vol / rétention urinaire

Usilay: marché, échanges

Ketik: décisions collectives, absentéisme aux réunions, interdits à lever

Certains ukin échappent à cette classification: ce sont ceux qui se transmettent à l'intérieur du ank, tels kasikenaku (lié à la croissance du riz et aux funćrailles) et usilaw (lié au double animal).

* Les détenteurs possèdent également le hochet-calebasse éseuey.

Le chiffre entre parenthèses indique le nombre de sanctuaires 
l'initiation pour conserver au village ses meilleurs lutteurs (l'initiation masculine ouvre la voie au mariage, état incompatible avec la pratique de la lutte); "attacher" la pluie en début d'hivernage lorsque, cette année-là, les jeunes et leurs aînés séjournent dans le bois des initiés.

Bien différent, nous l'avons signalé, est le champ relativement hétéroclite traité par les ukin "à harpon": protection, guérison, vol, accidents, meurtre, incendie, techniques de la forge, de la divination, enterrement, etc. Dans leur grande majorité, les maladies qu'ils provoquent attaquent les sens, les os, la peau, la "tête", les articulations, la locomotion, fonctions qui ne relèvent pas de l'ensemble bio-physiologique "interne" constitué, dans les représentations felup du corps, par la série asophage-poumons-caur-foie-intestins. Sans développer ici une question qui nous engagerait trop loin de l'objet de ce court article, nous noterons que les ukin "à harpon" visent préférentiellement ce qui, du corps, est directement investi dans les formes de la sociabilité quotidienne (ouïe, vue, parole, apparence extérieure, mobilité).

Que les affaires de la vie en société, avec les conflits qu'elles comportent, soient si minutieusement prises en charge par les ukin "à harpon", n'est pas sans rapport avec l'organisation sociale joola, de type segmentaire et acéphale, exempte de chefferies, de castes, de lamanat ${ }^{9}$. Ignorant les différences de statut liées à la naissance, l'aléa des rapports sociaux y est encore plus grand. Il faut tout à la fois l'autorité de puissances surnaturelles, et la possibilité humaine de diriger ces puissances pour organiser, régler ou apaiser "affaires", "discussions", histoires de vengeances et contre-vengeances qui courent de générations en générations, jalousies et suspicions, qui ne cessent de resurgir çà et là, tant il est vrai que l'égalité formelle en est le meilleur terreau. Rivalités d'appropriation sur les rizières ou les palmeraies, querelles de famille et rancours, ont été attisées par la guerre contre les Portugais. Pour tenter de désamorcer ces cycles de violence, certains se sont convertis. Mais tous restent extrêmement attentifs aux modes de gestion villageoise de tels conflits que, compte

${ }^{9}$ Du mot wolof lamane, "maître de la terre": cette institution est présente dans la plupart des sociétés paysannes sénégalaises. 
tenu de la distance d'avec les autorités étatiques, seuls les responsables d'ukin savent organiser. C'est à l'intérieur de ce champ, toujours mouvant, jamais "épuisé" - puisqu'au moment du décès il faudra encore y revenir ${ }^{10}$ - qu'apparaissent et se développent une série de pratiques magiques qui prennent comme supports des objets mobiliers (coques, cornes, etc.) ou fixes (le bèkin) que l'on peut ici qualifier de "fétiches". Dans ce domaine, la pratique magique n'est pas forcément liée à un objet matériellement repérable ${ }^{11}$ : prenons pour exemple le geste du kepuleenaku, qui consiste à souffler, en pulvérisant quelques gouttes de salive, ou délivrer d'une mauvaise parole. Lorsqu'il s'agit, pour un offenseur, de demander à la personne qu'il a offensée, de l'aider à lever la malédiction dont il est frappé, l'offensé mâche préalablement une noix de bukunumabu (tukuluna), dont il recrache les morceaux au visage de l'offenseur. Ce geste, effaçant de "la bouche dont elle est sortie" la première imprécation, suspend l' "affaire" jusqu'à ce qu'elle soit jugée publiquement. Le kepuleenaku simple est un gestz omniprésent dans la pratique cultuelle: ayant simplement valeur de bénédiction dans l'espace des ukin "à bâton", il acquiert un pouvoir spécifique lorsqu'il s'agit de traiter des dissensions entre individus, ou certaines maladies relevant des ukin "à harpon". Le kepuleenaku dans ce cas est typiquement un rituel de déliaison: déliaison de la parole imprécative, voire de l'action du bëkin. Ainsi, un voleur dont emotay, à la suite d'un sacrifice du plaignant, aurait lié les genoux, ira implorer un kepuleenaku aupress de l'amanen. Dans ce registre, bien d'autres pratiques restent à explorer: ainsi l'opération ewalisey, qui consiste, a l'insu des intéressés, à procéder à un transfert de forces de certains lutteurs vers celui dont les vieux ont décidé de maximiser les chances; ou encore le "travail" du magicien qui, lorsque les femmes partent

10 L'interrogatoire du mort n'est pas sans évoquer ce travail "d'épuisement de l'objet" dont nous parlent $M$. Ducornet et $M$. Guibal à propos d'objets modelés par un sujet au cours de la cure analytique (communication à l'URA 221, 11/4/1990).

11 A. de Surgy rappelle que chez les Evhé, les "charmes" bo peuvent se limiter à la connaissance d'une recette magique mettant en jeu des ingrédients, principalement végétaux, d'indispensables paroles magiques ( $g$ besa) parfois inintelligibles, et un rituel de préparation de quelque chose. 
pêcher au marigot, "écarte" les doubles aquatiques des habitants du village.

\section{Deux types de liens avec le bëkin}

Qu'en est-il, dans cette perspective, de la personne même de l'amanen? Les rituels d'acquisition d'un bëkin par le biais du ulungaw, nous l'avons signalé, sont fort différents des rituels d'intronisation aux ukin "à bâton". Ces derniers, à plus d'un titre, peuvent être considérés comme des funérailles anticipées: l'ancienne maison du nouveau responsable est cassée, les tantes paternelles apportent les pagnes funéraires qu'elles auraient remis à leur neveu le jour de sa mort, on chante les nyukulay qui exaltent les défunts... L'une des séquences des rituels d'intronisation retiendra ici notre attention: après être resté reclus cinq jours dans une hutte construite derrière sa maison, le nouvel initié couvert de la tête aux pieds d'un pagne noir, est emmené au bëkin où on le rase; puis on le couche à plat ventre, la tête audessus du trou où l'on verse le sang de l'animal sacrifié (une chèvre) mêlé au vin de palme, jusqu'à ce que ce mélange déborde. Lorsqu'il se releve, ses oncles maternels lui enduisent encore la tête de vin de palme, achevant sa transformation en véritable autel vivant ${ }^{12}$. De l'intronisé, on dit qu'"on l'a mis assis", entendu comme "on l'a fixé". Désormais mort à sa condition d'homme ordinaire (dès lors, "il ne connaît plus ses parents, sa femme, ses frères"), il devient obligé du bëkin ${ }^{13}$. La responsable de karaayaku (et, autrefois, les détenteurs des principaux ukin "à bâton"), est tenue à une abstinence sexuelle définitive. La maison du nouvel amanen sera reconstruite à proximité immédiate du sanctuaire.

Les rituels et les interdits qui s'appliquent au "roi" joola détenteur de këyëku, auquel on s'adresse d'ailleurs comme au bëkin lui-même

${ }^{12}$ Ce rituel est abondamment décrit par N. Ukeyeng Diatta (1979).

13 Cette transformation profonde de la personne de l'intronisant n'est pas sans évoquer le procès de "déparentalisation" qui précède la métamorphose du chef initié gourmantché (cf. M. Cartry, 1987: 131-239). 
("maan..."), offrent la figure paradigmatique de l'incorporation de l'amanten à son bëkin. Placé au sommet de la hiérarchie religieuse, këyëku est le garant de toutes les disjonctions opérées entre les sexes, les saisons, les substances (eau et vin de palme par exemple, qu'il est interdit de mélanger), en même temps qu'il médiatise le passage des un(e)s aux autres. Son desservant, incarnation même de l'essence du ninii, de la séparation et des règles d'incompatibilité fondées sur les dangers d'un "cumul de l'identique"14, est soumis en permanence aux interdits qui visent périodiquement hommes (lors de l'initiation) ou femmes ordinaires (lors des périodes menstruelles ou de l'accouchement). Ainsi ne peut-il voir ou approcher la mer, cultiver ou traverser les rizières, franchir une rivière, être surpris à boire ou manger, pénétrer une autre maison que la sienne, être mouillé par la pluie, etc.

De leur relation au bëkin, les détenteurs "à harpon", certes, ne sortent pas moins indemnes. Un manquement de leur part aux règles du sanctuaire et du rituel sacrificiel, un mauvais usage de leur pouvoir, cupidité ou négligence dans l'accomplissement de leurs fonctions, seront chèrement payés. Qu'éclatent par exemple de sanglantes bagarres a l'occasion des luttes villageoises, le responsable du bëkin tutélaire devra aussitôt immoler du gros bétail prélevé sur son propre avoir. S'il est sorcier lui-même, le bëkin, dit-on, le tuera rapidement. Libre à eux cependant d'orienter l'énergie de leur bëkin dans telle direction, d'attaquer, à la demande d'un sacrifiant, telle ou telle personne, d'interpréter les demandes et prescriptions que leur transmet, par voie de rêve ou de divination, le génie qu'ils ont "planté". Les détenteurs du harpon peuvent pratiquer le buyuebu (sacrifier contre quelqu'un que je soupçonne de m'avoir fait du mal, ou de m'avoir volé), dont les aînés disent eux-mêmes: "nous qui vous avons mis au monde, nous avons peur de faire buyuèbu. Buyuèbu finit un hank" (tue jusqu'au dernier tous les membres d'un segment de lignage). On remarquera que les sanctuaires "à harpon" sont toujours aménagés dan; la maison ou la cour de l'amañen; il peut s'agir d'une petite chambrie ou d'une petite case édifiée lors du ulangaw, ou de grands sanctuaires,

${ }^{14}$ Cf. F. Héritier, 1979: 209-244. 
tel bulampanabu, qui survivent à la mort de leur détenteur et sont alors transportés dans la maison du nouveau propriétaire.

Tandis que les ukin "à bâton" sont immuablement fixés à l'endroit même de leur fondation (au changement de responsable, on ne fait qu'inverser l'orientation du sanctuaire, ou plus exactement de l'aire réservée à l'assistance), les ukin "à harpon" suivent leur détenteur, allant - cas rare au demeurant - jusqu'à nomadiser avec lui. Tel est l'exemple de ce féticheur errant, ayant fui le village où il a perdu parents, femmes et enfants. Sans résidence fixe, il transporte avec lui son bëkin ankurenaw, réduit à sa plus simple expression: la coquille de libation kasendaku, et une corde sur laquelle est fixée, par l'intermédiaire de quatre grelots, une corne de gazelle obturée. Cet instrument divinatoire (en jouant sur la tension de la corde tendue entre le pouce gauche et le gros orteil, on fait tourner la corne) lui sert d'autel, lorsqu'avant chaque consultation il y sacrifie un poulet ou du vin de palme.

A la mort de l'amañen, les ukin plantés individuellement sont détruits. Dans les rares autres cas, ils sont "replantés" au cours d'un jour et d'une nuit d'activité rituelle intense.

Quel que soit le sanctuaire, le harpon fera l'objet, au cours des funérailles, d'un rituel de détache d'avec son propriétaire: añanorol, "le séparer". Dans l'après-midi qui suit le décès, sur la place où est exposé le défunt ont lieu les danses publiques de nyukulay: elles sont interrompues un court instant par l'arrivée des porteurs de harpon et de hochets-calebasse; l'un d'eux s'arrête face à l'estrade du mort et balance le harpon du défunt en direction des quatre points cardinaux, avant de le remporter dans le bëkin. Aucun rituel de cet ordre ne sera effectué sur le bâton.

Au regard des contraintes d'usage dont il est l'objet, tout porte à penser que le harpon sert en quelque sorte de doublet au corps de l'amañen, préservant ce dernier des dangers et des contraintes qu'impose une trop grande intimité avec le bëkin. En canalisant la puissance de ce dernier, le harpon opère tout à la fois la coupure et la jonction entre le bëkin et son détenteur.

De nous être arrêtés un instant sur ces objets et les modes d'installation de l'amañen ou, selon les cas, d'acquisition d'un bëkin, 
nous a permis d'esquisser une ligne de démarcation entre deux modèles: médiatisation et dédoublement du rapport au bëkin d'une part, inféodation et incorporation du bëkin de l'autre. Si, dès leur installation, les détenteurs du harpon engagent leur avoir pour acquérir et conserver ce pouvoir d'orienter la force de leurs ukin, c'est au prix d'une dépossession de soi, d'une transformation radicale de leur être, que se noue la relation particulière des desservants "à bâton" à leur bekkin. Nous pourrions en partie reprendre ici les termes de la différenciation proposés par A. de Surgy à propos des faits évhé: "... par opposition à un prêtre ayant été "saisi" par une divinité ou à tout autre intermédiaire entre les hommes et les invisibles puissances qui l'environnent, l'homme méritant le titre de féticheur négocie moins avec des esprits qu'il ne réussit à les dominer, et, contrairement aux actes d'adoration, d'adulation ou de prière, les actes fétichistes sont de nature à produire immédiatement ou quasi-automatiquement leurs effets", à cette réserve près que la pratique des desservants "à bâton" ne comporte guère d'adoration ou d'adulation. Plus qu'à leurs actes, c'est à leur position dans la sphère sacrificielle que l'on se référerait pour définir leur statut. Brutalement inauguré au cours des rituels d'intronisation, un processus d'altération irréversible de leur être les a consacrés au bëkin, tout à tour comme autel, sacrificateur obligé, et victime: de l'amañen à son bëkin, le rapport intime initié par la mise en scène d'une mort anticipée va se conclure, en cas de sénilité ou de maladie grave, par une mise à mort réelle: les responsables adjoints du sanctuaire fendront le crâne à coups de hache, ou briseront la colonne vertébrale de l'amañen déficient.

Revenons au bëkin: voilà un lieu, un objet, une force..., selon l'angle sous lequel on le considère, qui, à l'instar de la plupart des matériaux présentés dans le cadre du séminaire de l'URA 221 sur la notion de fétiche, déjoue les oppositions classiques : "fabrique"/ "naturel"; "magique"/"religieux"; "maléfique"/"bénéfique"; "contenant"/ "contenu"; "divinités"/"fétiches", etc. Quoiqu'immobile, fixé, le bëkin, autel ou sanctuaire, devient, au fur et à mesure des sacrifices, un lieu vivant, arrosé et nourri de sang, de vin de palme, de grains ou de 
farine de riz, toujours en transformation ${ }^{15}$ par "absorption" d'éléments fabriqués (poteries, morceaux de fer) ou "naturels". Il se constitue dans un processus d'accumulation permanente d'objets minéraux (coquilles, outils ou armes de fer), animaux (mâchoires, cornes), végétaux (arbres plantés dans le bëkin), organiques (placentas, excréments) qui tous ont la particularité d'avoir été fortement liés à la personne du sacrifiant. Cependant, au risque de durcir provisoirement l'opposition sur laquelle nous nous sommes arrêtée, il apparaît que dans l'espace des ukin, deux attitudes, deux types de liens caxistent: d'un côté, manipulation "à la demande" d'objets, médiation et dédoublement de la force du bekkin et du corps de l'amanen, pratique de type fétichiste, de l'autre, intimité et immanence de la relation au bëkin, transformation de l'amañen en "être sacrificiel" 16 plutôt qu'en féticheur.

\section{O. Journet}

URA 221, EPHE-CNRS

(Septembre 1991)

${ }^{15}$ Comme le met en évidence $\mathbf{M}$. Coquet à propos du didiro bwaba, c'est l'une des caractéristiques structurelles du fétiche.

${ }^{16} \mathrm{Au}$ sens développé par A. Zempléni, à propos de l'altération liturgique du possédé, comme de la victime animale (1987: 267-317). 


\section{Références bibliographiques}

Cartry, M.

1987 "Le suaire du chef", Sous le masque de l'animal. Essais sur le sacrifice en Afrique Noire, Paris, PUF, 131-239.

Coquet, $\mathrm{M}$.

1987 "Une esthétique du fétiche", Systèmes de pensée en Afrique Noire, VIII, Fétiches. Objets enchantês, mots réalisés, Paris, EPHECNRS, 111-138.

Diatta, Ukeyeng N.

1979 "Le taureau symbole de mort et de vie dans l'initiation de la circoncision chez les Diola", Mémoire EHESS, Paris.

Henry, Ch.

1991 Rapports d'âge et de sexe chez les Bijogo (Guinée-Bissau), Thèse de doctorat, Université de Paris X, 312 p.

Héritier, Fr.

1979 "Symbolique de l'inceste et de sa prohibition", in La fonction symbolique, NRF, 209-244.

Journet, $\mathrm{O}$.

1987 "Le sang des femmes et le sacrifice: l'exemple joola". Sous le masque de l'animal. Essais sur le sacrifice en Afrique Noire. Paris, PUF, 241-266.

Sapir, J. David

1977 "Fecal animals: an example of complementary totemism", Man, XII, 1-21.

Surgy, A. de

1987 "Examen critique de la notion de fétiche à partir du cas évhé (Togo)", Systèmes de Pensée en Afrique noire, VIII, Fétiches. Objets enchantés, mots réalisés, Paris, EPHE-CNRS, 263-303.

Thomas, L.V.

1959 Les Diola. Essai d'analyse fonctionnelle sur une population de Basse-Casamance, IFAN, Dakar, 821 p.

Zempléni, A.

1987 "Des êtres sacrificiels", Sous le masque de l'animal. Essais sur le sacrifice en Afrique Noire, Paris, PUF, 267-317. 